\title{
The van der Waals equation: analytical and approximate solutions
}

\author{
Mario N. Berberan-Santos \\ Centro de Química-Fisica Molecular, Instituto Superior Tecnico, Lisboa P-1049-001, Portugal \\ E-mail: berberan@mail.ist.utl.pt \\ Evgeny N. Bodunov \\ Department of Physics, Petersburg State Transport University, St. Petersburg 190031, Russia \\ E-mail: evgeny.bodunov@rol.ru \\ Lionello Pogliani* \\ Dipartimento di Chimica, Università della Calabria, via P. Bucci, 14/C, Rende CS I-87036, Italy \\ E-mail: lionp@unical.it
}

Received 8 February 2007; Revised 23 February 2007

\begin{abstract}
The thermodynamic properties, enthalpy of vaporization, entropy, Helmholtz function, Gibbs function, but especially the heat capacity at constant volume of a van der Waals gas (and liquid) at the phase transition are examined in two different limit approximations. The first limit approximation is at the near-critical temperatures, i.e., for $T / T_{c} \rightarrow 1$, where $T_{c}$ is the critical temperature, the other limit approximation is at the near-zero temperatures, $T \rightarrow 0$. In these limits, the analytical equations for liquid and gas concentrations at saturated conditions were obtained. Although the heat capacities at constant volume of a van der Waals gas and liquid do not depend on the volume, they have different values and their change during the phase transition was calculated. It should be noticed that for real substances the equations obtained at the nearzero temperature are only valid for $T>T_{\text {triple point }}$ and $T \ll T_{c}$, which means that found equations can be used only for substances with $T_{\text {triple point }} \ll T_{c}$.
\end{abstract}

KEY WORDS: thermodynamics, van der Waals equation, phase transition, heat capacities, critical temperature

\section{Introduction}

Many applications of thermodynamics are concerned with the behavior of fluids and gases. Thermodynamic properties, such as internal energy and enthalpy from which one calculates the heat and work requirements of industrial

\footnotetext{
* Corresponding author.
} 
processes are not readily measurable. In fact nobody has ever heard of energymeter, enthalpymeter or entropymeter. They can, however, be calculated from volumetric data using an appropriate equation of state (EOS).

Consider first an ideal gas (also called perfect gas). It is a collection of a very large number of individual molecules that do not exert forces upon each other (except by elastic collisions) and which are so small that they can be treated as if they were zero-volume massless points. This picture is adequate to describe the behavior of real gases only at very low pressures. The EOS for 1 mole of an ideal gas is,

$$
P V=R T .
$$

Here, $P$ is the gas pressure, $V$ is the molar volume, $T$ is the temperature, and $R$ is the gas constant.

To obtain a more realistic EOS, van der Waals introduced corrections that account for the finite volumes of the molecules and for the attractive forces (van der Waals forces) they exert upon each other at sufficiently small distances. In each mole of gas there is a volume $(V-b)$ available for the free motion that is somewhat less than the total volume. The term $b$ is the excluded volume of the particles per mole (sometimes called co-volume). The excluded volume is representative of the repulsion forces among the molecules of the gas. These forces induce a volume larger than the actual volume of the molecules, because in their movement they do not truly touch each other.

To take into account the forces of attraction, van der Waals considered that molecules in the bulk of gas are equally attracted in all directions by the surrounding molecules, even if this is not the case for the molecules next to the wall of the container. These molecules experience a net inward force and thus they are decelerated when traveling in the direction of the wall. If we recall that the kinetic theory of gases postulates that pressure is the number of collisions per unit area and per unit time, this inward force results in fewer molecules hitting the wall, that is, the pressure observed in a real gas must be smaller than the pressure present in an ideal gas. The reduction in pressure will be proportional to the number of molecules per unit volume, $N_{A} / V\left(N_{A}\right.$ is Avogadro number), in the layer next to the wall, and to the number of molecules per unit volume in the next layer, being their interactions attractive in character. Therefore, the reduction in pressure, $\Delta P=P^{\prime}-P\left(P^{\prime}\right.$ is pressure calculated from simple kinetic theory, known as the internal pressure of the gas), is: $\Delta P \propto\left(N_{A} / V\right)^{2}$, or $P^{\prime}=P+a / V^{2}$. Here $a$ (together with $b$ ) is a van der Waals constant. Taking both corrections into account leads to the well-known van der Waals equation of state (WEOS)

$$
\left(P+\frac{a}{V^{2}}\right)(V-b)=R T .
$$


The terms $a$ and $b$ are characteristic of each gas and can be obtained by analyzing the WEOS at the critical point, i.e., zeroing the first and second derivative, $\mathrm{d} P / \mathrm{d} V$ and $\mathrm{d}^{2} P / \mathrm{d} V^{2}$, at this point, where the $P-V$ curve has a flat inflection,

$$
P_{c}=\frac{a}{27 b^{2}}, \quad V_{c}=3 b, \quad T_{c}=\frac{8 a}{27 R b}, \quad z_{c}=\frac{3}{8} .
$$

Here, $P_{c}, V_{c}$, and $T_{c}$ are the critical values for pressure, molar volume, and temperature, and $z_{c}$ is the compressibility $(=P V / R T)$ at the critical point.

WEOS (together with Maxwell's principle which removes in the van der Waals equation regions where $\left.(\partial P / \partial V)_{T}>0\right)$ represents qualitatively the behavior of the real gas over the whole range of gas, vapor, and liquid. It does not give the numerical details correctly, yet it never leads to physical non-sense, it is a relatively simple equation, and it is widely used to illustrate the general behavior of non-ideal gases. Other two-parameter EOS exist [1, 2], but WEOS continues to enjoy great popularity because of the relative simplicity and very simple ideas used. WEOS is even able to predict negative pressures, but this is another story.

If the analytical solution of the van der Waals equation is known, one can calculate the different thermodynamic properties of real gas.

\section{Enthalpy of vaporization $L_{v}$}

The Clausius-Clapeyron equation for the saturation vapor pressure $P_{\text {sat }}$ gives

$$
\frac{\mathrm{d} P_{\text {sat }}}{\mathrm{d} T}=\frac{L_{v}}{T\left(V_{G}^{*}-V_{L}^{*}\right)} .
$$

Here, $V_{G}^{*}$ and $V_{L}^{*}$ are the molar volumes of gas and liquid under saturation condition, respectively. Equation (4a) can be solved for $L_{v}$, to obtain,

$$
L_{v}=T\left(V_{G}^{*}-V_{L}^{*}\right) \frac{\mathrm{d} P_{\mathrm{sat}}}{\mathrm{d} T} .
$$

\section{Entropy, $S$}

An equation connecting the entropy $S$ and the pressure is obtained from the equation for the free energy $F(F=U-T S$, where $U$ is the internal energy of a gas; for a mnemonic method to derive and check many thermodynamic equations see [3] and references therein)

$$
\mathrm{d} F=-S \mathrm{~d} T-P \mathrm{~d} V .
$$


Now, by the help of one of the Maxwell equations, i.e., $\left(\frac{\partial S}{\partial V}\right)_{T}=\left(\frac{\partial P}{\partial T}\right)_{V}$, we obtain the entropy if the pressure is known:

$$
S(T, V)-S\left(T, V_{1}\right)=\int_{V_{1}}^{V}\left(\frac{\partial P}{\partial T}\right)_{V} \mathrm{~d} V
$$

The particular solution shown in equation (6b), can be obtained if we consider that at the saturated conditions, $P_{\text {sat }}$ does not depend on $V$, which allows writing: $S(T, V)-S\left(T, V_{1}\right)=\frac{\mathrm{d} P_{\text {sat }}}{\mathrm{d} T}\left(V-V_{1}\right)$, where $V_{L} \leqslant V, V_{1} \leqslant V_{G}$, which with equation (4b) obtains,

$$
S_{\mathrm{sat}}\left(T, V_{G}^{*}\right)-S\left(T, V_{L}^{*}\right)=\frac{\mathrm{d} P_{\mathrm{sat}}}{\mathrm{d} T}\left(V_{G}^{*}-V_{L}^{*}\right)=\frac{L_{v}}{T} .
$$

\section{Internal energy, $U$}

The fundamental equation of thermodynamics, which combines the first and the second law of thermodynamics reads

$$
T \mathrm{~d} S=\mathrm{d} U+P \mathrm{~d} V
$$

Another important equation is known as the $T \mathrm{~d} S$ equation (there are two of them), which can be derived starting with the exact differential of $S=S(T, V)$, multiplying both sides by $T$ and using the Maxwell equation, $\left(\frac{\partial S}{\partial V}\right)_{T}=\left(\frac{\partial P}{\partial T}\right)_{V}$, reminding that $C_{V}=T(\partial S / \partial T)_{V}$ is the heat capacity at constant volume, [4]

$$
T \mathrm{~d} S=C_{V} \mathrm{~d} T+T\left(\frac{\partial P}{\partial T}\right)_{V} \mathrm{~d} V
$$

Combining equations (7) and (8) we get (see for example [4])

$$
\mathrm{d} U=C_{V} \mathrm{~d} T+\left[T\left(\frac{\partial P}{\partial T}\right)_{V}-P\right] \mathrm{d} V .
$$

For a van der Waals gas we get,

$$
\mathrm{d} U=C_{V} \mathrm{~d} T+\frac{a}{V^{2}} \mathrm{~d} V
$$

This means that, $\left(\frac{\partial U}{\partial V}\right)_{T}=\frac{a}{V^{2}}$. This last expression plays a major role in thermodynamics, where it is usually denoted as the isothermal coefficient $\boldsymbol{\pi}_{T}$, which equals zero for an ideal (or perfect) gas. From equation (10) we can also obtain, $\left(\frac{\partial U}{\partial T}\right)_{V}=C_{V}(T, V)$. Equation (10) and the derived relations are not valid for the saturated vapor, even if they are valid for metastable states: oversaturated vapor 
and stretched liquids. In fact, at saturated conditions, WEOS is not described by equation (2). At saturated conditions (see equation (9)),

$$
\mathrm{d} U_{\text {sat }}=C_{\text {sat }, V} \mathrm{~d} T+\left[T \frac{\mathrm{d} P_{\text {sat }}}{\mathrm{d} T}-P_{\text {sat }}(T)\right] \mathrm{d} V,
$$

where $C_{\mathrm{sat}, V}$ is the heat capacity of the system liquid plus saturated gas at constant volume and at saturated conditions. In particular,

$$
U_{\text {sat }}\left(T, V_{G}^{*}\right)-U_{\text {sat }}\left(T, V_{L}^{*}\right)=\left[T \frac{\mathrm{d} P_{\text {sat }}}{\mathrm{d} T}-P_{\text {sat }}(T)\right]\left(V_{G}^{*}-V_{L}^{*}\right) .
$$

Combining this equation with equation (6b) we have

$$
U_{\text {sat }}\left(T, V_{G}^{*}\right)-U_{\text {sat }}\left(T, V_{L}^{*}\right)=L_{v}-P_{\text {sat }}(T)\left(V_{G}^{*}-V_{L}^{*}\right) .
$$

Equations (6b) and (12b) follow from equation (4a) if it is reminded that in processes where $T$ and $P$ are constants: $L_{v}=\Delta Q=T \Delta S=\Delta U+P_{\text {sat }} \Delta V$.

\section{Free energy $F$}

From equation (5), in processes where $T$ is constant, it follows that: $\left(\frac{\partial F}{\partial V}\right)_{T}=-P(T, V)$

This equation allows calculating $F$ if the pressure is known, through equation,

$$
F(T, V)-F\left(T, V_{1}\right)=-\int_{V_{1}}^{V} P(T, V) \mathrm{d} V .
$$

As, at the saturated conditions, $P_{\text {sat }}$ does not depend on $V$, then $F_{\text {sat }}(T, V)-$ $F_{\text {sat }}\left(T, V_{1}\right)=-P_{\text {sat }}(T)\left(V-V_{1}\right)$, and, in particular,

$$
F_{\text {sat }}\left(T, V_{G}^{*}\right)-F_{\text {sat }}\left(T, V_{L}^{*}\right)=-P_{\text {sat }}(T)\left(V_{G}^{*}-V_{L}^{*}\right) .
$$

\section{Gibbs function, $G$}

$$
G(T, V)=F+P(T, V) V .
$$

At the saturated conditions, the Gibbs function, $G_{\text {sat }}(T, V)=F_{\text {sat }}(T, V)+$ $P_{\text {sat }}(T) V$, does not change when the volume is changing and the temperature is kept constant, i.e., $G_{\mathrm{sat}}(T, V)-G_{\mathrm{sat}}\left(T, V_{1}\right)=0$. In particular, $G_{\text {sat }}\left(T, V_{G}^{*}\right)-$ $G_{\text {sat }}\left(T, V_{L}^{*}\right)=0$. 


\section{Heat capacity at constant volume, $C_{V}$}

Applying the condition that equation (5) is a perfect differential (the second derivative of the entropy does not depend on the order of differentiation, $\left.\partial^{2} S / \partial T \partial V=\partial^{2} S / \partial V \partial T\right)$, we get [reminding that, $C_{V} / T=(\partial S / \partial T)_{V}$, and $(\partial S / \partial V)_{T}=(\partial P / \partial T)_{V}($ a Maxwell relation $\left.)\right]$

$$
\left(\frac{\partial C_{V}}{\partial V}\right)_{T}=T\left(\frac{\partial^{2} P}{\partial T^{2}}\right)_{V} .
$$

The van der Waals equation predicts that the pressure is a linear function of the temperature, therefore $\left(\partial C_{V} / \partial V\right)_{T}=0$ and $C_{V}$ does not depend on the gas volume. As, at very large volumes, the van der Waals gas behaves like an ideal gas, the heat capacity of the van der Waals gas equals the heat capacity of an ideal gas, a fact that contradicts the experimental evidence [5]. This conclusion is not valid for a saturated vapor, whose pressure depends on the temperature in a more complicated way (see below). By the same reasons the heat capacity of a $\mathrm{vdW}$ fluid does not depend on the volume. The difference between heat capacities of a van der Waals gas and a van der Waals fluid is obtained below.

The heat capacities at the constant pressure and constant volume are connected by the equation,

$$
C_{P}=C_{V}-T\left(\frac{\partial P}{\partial T}\right)_{V}^{2} /\left(\frac{\partial P}{\partial V}\right)_{T} .
$$

\section{Discussion}

The last equation shows that $C_{P}>C_{V}$ because $(\partial P / \partial V)_{T}<0$. In fact, while the volume decreases, the pressure increases in all systems. The simpler analytical solution for the pressure of a van der Waals gas and liquid tells that,

$$
P=\frac{R T}{V-b}-\frac{a}{V^{2}} .
$$

This equation allows to obtain

$$
\left(\frac{\partial P}{\partial V}\right)_{T}=-\frac{R T}{(V-b)^{2}}+\frac{2 a}{V^{3}}<0 .
$$

For gases (gas volume) $V_{G} \gg b$, and inequality (18) gives $V_{G}>2 a / R T$. Taking into account equation (3) we obtain, $V_{G}>9 T_{c} V_{c} / 4 T=9 V_{c} / 4 T_{r}$, which means that at low temperatures, the gas volume, $V_{G}$, must be large enough, and the gas phase is the only phase of the system. From now on the following dimensionless reduced volume, temperature, and pressure will be used: $V_{r}=V / V_{c}$, and $T_{r}=T / T_{c}, P_{r}=P / P_{c}$. At smaller volumes, saturated vapor, and liquid coexist. 
Now, by the aid of equations (16) and (17), and also by the aid of the last result on $V_{G}$, the following interesting expression for the heat capacity of a van der Waals gas is obtained.

$$
C_{P}-C_{V}=\frac{R}{1-V_{G} / V}=\frac{R}{1-\frac{9}{4 T_{r} V_{r}}} .
$$

This expression shows that for a van der Waals gas: $C_{P}-C_{V}>R$. This difference increases with decreasing gas volume.

For liquids at low temperature, $V_{L}>b$ and $V_{L} \sim b$, and by the aid of equation (18) we get,

$$
-\frac{R T}{(V-b)^{2}}+\frac{2 a}{b^{3}}<0
$$

From this equation, and by the aid of equations (3), i.e., $b=V_{c} / 3$, and $R b / 2 a=4 / 27 T_{c}$, and reminding that $V_{L} \geqslant b$, the following result is obtained

$$
\frac{V_{c}}{3} \leqslant V_{L}<\frac{V_{c}}{3}\left(1+\frac{2}{3 \sqrt{3}} \sqrt{T_{r}}\right) .
$$

This result tells that at low temperatures, the volume of the liquid, $V_{L}$, is small enough. At larger volumes, saturated vapor, and liquid can coexist.

Equation (16) with equations (17) and (21) allow the estimation of the heat capacity for the liquid,

$$
C_{P}-C_{V}=\frac{R}{1-\frac{9 \times 27}{4 T_{r}}\left(V_{r}-1 / 3\right)^{2}},
$$

which tells that also for a van der Waals liquid $C_{P}-C_{V}>R$, and that this difference increases with increasing liquid volume.

For intermediate volumes, where liquid and saturated gas coexist, the volume, $V$, occupied by the system obeys the following relation,

$$
\frac{V_{c}}{3}+\frac{2 V_{c}}{9 \sqrt{3}} \sqrt{T_{r}}<V<\frac{9}{4} V_{c} / T_{r}
$$

Equation (16) is not valid for saturated conditions where pressure does not depend on the volume, i.e., here we are facing a first-order phase transition (following Ehrenfest's classification) for which $C_{P} \rightarrow \infty$.

While the analytical solution for pressure of a van der Waals liquid and gas is very simple (see equation 17), solutions for the vapor pressure and molar volumes of gas and liquid under saturated conditions (at the liquid-vapor boundary, $P_{\text {sat }}, V_{G}^{*}$, and $V_{L}^{*}$ ) are usually obtained numerically $[6,7]$. Analytical solutions for these values are known in very limited regions of temperatures [8]. 
In this paper, we obtained these solutions in the whole region of coexistence of saturated-vapor and liquid, i.e., for temperatures from $0 \mathrm{~K}$ to $T_{c}$. Equation (17) can be rewritten, using the reduced concentration, $c=1 / V_{r}$ (note that $C=1 / V$ has the meaning of molar concentration), reduced pressure, and reduced temperature into the following form,

$$
P_{r}=\frac{8 T_{r}}{3 / c-1}-3 c^{2}
$$

By the aid of the Maxwell rule and WEOS, it is possible to obtain the equations for reduced liquid and gas concentrations at the gas-liquid boundary, $c_{L}^{*}$ and $c_{G}^{*}$, as well as the reduced saturated-vapor pressure, $P_{\mathrm{sat}, r}$, and the reduced temperature, $T_{r},[6,7]$

$$
\begin{gathered}
\ln \frac{3 / c_{G}^{*}-1}{3 / c_{L}^{*}-1}=\frac{c_{L}^{*}-c_{G}^{*}}{c_{L}^{*}+c_{G}^{*}}\left(\frac{3}{3-c_{L}^{*}}+\frac{3}{3-c_{G}^{*}}\right), \\
P_{\mathrm{sat}, r}=c_{G}^{*} c_{L}^{*}\left\lfloor 3-\left(c_{G}^{*}+c_{L}^{*}\right)\right\rfloor, \\
T_{r}=\frac{1}{8}\left(c_{G}^{*}+c_{L}^{*}\right)\left(3-c_{G}^{*}\right)\left(3-c_{L}^{*}\right) .
\end{gathered}
$$

Note that the molar volumes in the liquid and gas states at the gas-liquid boundary are

$$
V_{L}^{*}=3 b / c_{L}^{*}, \quad V_{G}^{*}=3 b / c_{G}^{*} .
$$

The detailed calculations for $c_{G}^{*}$ and $c_{L}^{*}$ are displayed in Appendix A. The solutions at the near-critical temperatures, i.e., for $T_{r} \rightarrow 1, c_{L}^{*} \rightarrow 1$, and $c_{G}^{*} \rightarrow 1$, for $c_{G}^{*}$ and $c_{L}^{*}$ are the following,

$$
\begin{gathered}
c_{L}^{*}=1+2\left(1-T_{r}\right)^{1 / 2}+\frac{2}{5}\left(1-T_{r}\right)-\frac{13}{25}\left(1-T_{r}\right)^{3 / 2}+0.115\left(1-T_{r}\right)^{2}, \\
c_{G}^{*}=1-2\left(1-T_{r}\right)^{1 / 2}+\frac{2}{5}\left(1-T_{r}\right)+\frac{13}{25}\left(1-T_{r}\right)^{3 / 2}+0.207\left(1-T_{r}\right)^{2}, \\
\frac{1}{2}\left(c_{L}^{*}+c_{G}^{*}\right)=1+\frac{2}{5}\left(1-T_{r}\right)+0.161\left(1-T_{r}\right)^{2} .
\end{gathered}
$$

where equation (29) is valid for $0 \leqslant T_{r} \leqslant 1$ (see figure 1 , dotted line), equation (30) is, instead, valid for $0.46<T_{r} \leqslant 1$ (see figure 2 , dotted line), and equation (31) is valid for $0.25<T_{r} \leqslant 1$ (see figure 3 , dotted line). 


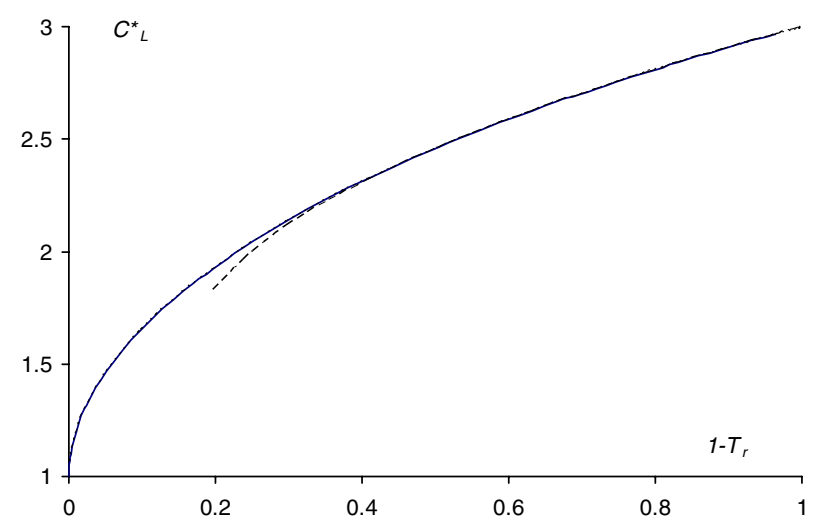

Figure 1. Dependence of the reduced liquid concentration, $C_{L}^{*}$, on the reduced temperature, $T_{r}$ : exact numerical solution (solid line), equation (29) (near-critical temperature limit, dotted line), and equation (32) (low-temperature limit, dashed line). Solid and dotted lines are practically coincident (in the limits of figure precision).

Appendix B will show that at the near-zero temperatures, $T_{r} \rightarrow 0, c_{L}^{*} \rightarrow 3$, $c_{G}^{*} \rightarrow 0:$

$$
\begin{gathered}
c_{L}^{*}=\frac{3}{2}\left(1+\sqrt{1-\frac{32}{27} T_{r}}\right), \\
c_{G}^{*}=\frac{3 c_{L}^{*}}{\mathrm{e}\left(3-c_{L}^{*}\right)} \exp \left(-\frac{1}{1-c_{L}^{*} / 3}\right), \\
\frac{1}{2}\left(c_{L}^{*}+c_{G}^{*}\right)=\frac{3}{2}-\frac{4}{9} T_{r}-0.15 T_{r}^{2} .
\end{gathered}
$$

Equation (32) is valid for $0 \leqslant T_{r}<0.7$ (see figure 1 , dashed line), equation (33) is, instead, valid for $0 \leqslant T_{r}<0.33$ (see figure 2 , dashed line), and equation (34) is valid for $0 \leqslant T_{r}<0.64$ (see figure 3 , dashed line). These results are obtained from the fitting procedure. Equations (29)-(34) together with equations (26) and (28) allow calculating the change of thermodynamic functions at saturated conditions. Note that the values of the thermodynamic functions depend on the combination of two products $\left(V_{G}^{*}-V_{L}^{*}\right) \mathrm{d} P_{\text {sat }} / \mathrm{d} T$ and $P_{\text {sat }}(T)\left(V_{G}^{*}-V_{L}^{*}\right)$. Taking into account equations (26) and (28), we have,

$$
P_{\text {sat }}\left(V_{G}^{*}-V_{L}^{*}\right)=3 b P_{c} P_{r}\left(\frac{1}{c_{G}^{*}}-\frac{1}{c_{L}^{*}}\right)=3 b P_{c}\left(3-c_{G}^{*}-c_{L}^{*}\right)\left(c_{L}^{*}-c_{G}^{*}\right) .
$$




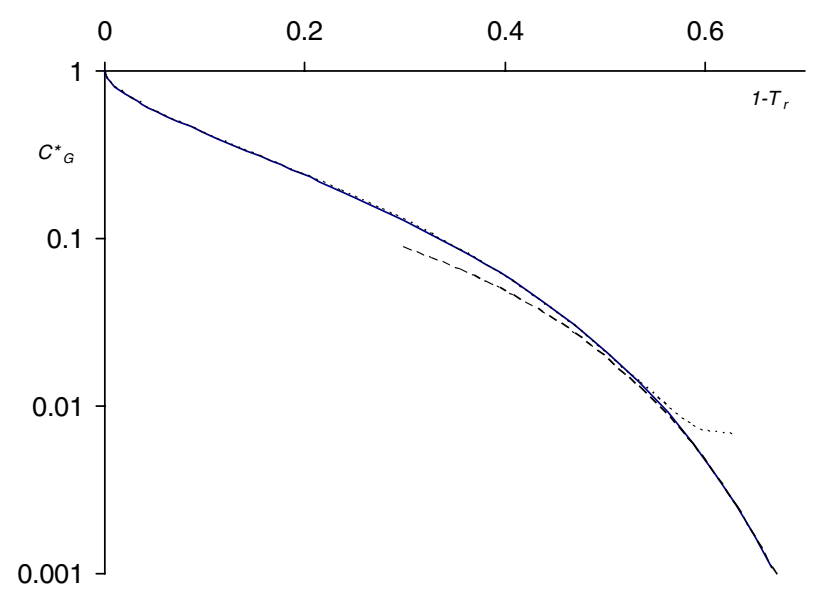

Figure 2. Dependence of the reduced gas concentration, $C_{G}^{*}$, on the reduced temperature, $T_{r}$ : exact numerical solution (solid line), equation (30) (near-critical temperature limit, dotted line), and equation (33) (near-critical temperature limit, dashed line).

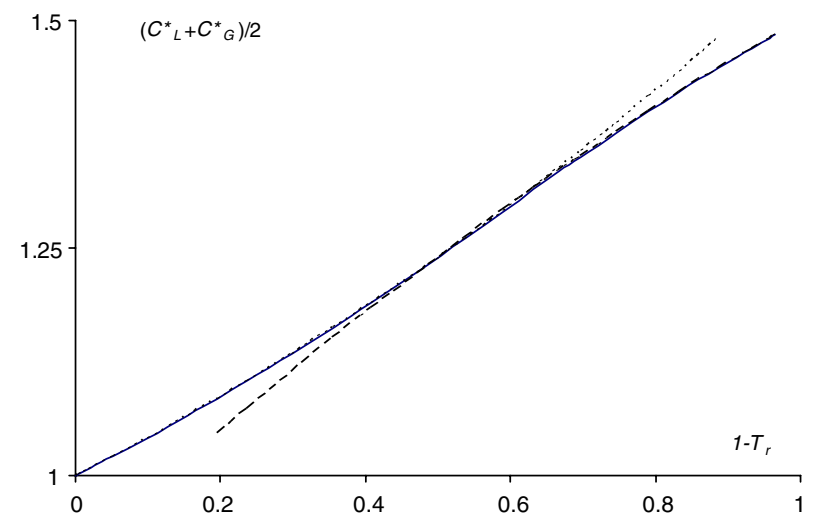

Figure 3. Dependence of $\left(C_{G}^{*}+C_{L}^{*}\right) / 2$ on the reduced temperature, $T_{r}$ : exact numerical solution (solid line), equation (31) (near-critical temperature limit, dotted line), and equation (34) (nearcritical temperature limit, dashed line).

\subsection{Thermodynamic functions at near-critical temperature}

At the near-critical temperatures, equations (35), (29), and (30) give

$$
P_{\text {sat }}\left(V_{G}^{*}-V_{L}^{*}\right)=12 b P_{c}\left(1-T_{r}\right)^{1 / 2}-\frac{318 b P_{c}}{25}\left(1-T_{r}\right)^{3 / 2}+O\left[\left(1-T_{r}\right)^{2}\right] .
$$


For the big $O$-notation see note. ${ }^{1}$ Note that $b P_{c}=R T_{c} / 8$ according to equation (3). Therefore, equation (36a) can be rewritten as

$$
P_{\text {sat }}\left(V_{G}^{*}-V_{L}^{*}\right)=\frac{3}{2} R T_{c}\left(1-T_{r}\right)^{1 / 2}-\frac{318 R T_{C}}{25 \times 8}\left(1-T_{r}\right)^{3 / 2}+O\left[\left(1-T_{r}\right)^{2}\right] .
$$

At the same temperatures,

$$
\begin{gathered}
P_{\mathrm{sat}, r}=1-4\left(1-T_{r}\right)+4.8\left(1-T_{r}\right)^{2}, \quad 0.46<T_{r} \leqslant 1, \\
\frac{1}{c_{G}^{*}}-\frac{1}{c_{L}^{*}}=4\left(1-T_{r}\right)^{1 / 2}+\frac{194}{25}\left(1-T_{r}\right)^{3 / 2}+O\left[\left(1-T_{r}\right)^{2}\right]
\end{gathered}
$$

and

$$
\frac{\mathrm{d} P_{\mathrm{sat}, r}}{\mathrm{~d} T_{r}}=4-9.6\left(1-T_{r}\right) .
$$

Thus, the other product,

$$
\left(V_{G}^{*}-V_{L}^{*}\right) \frac{\mathrm{d} P_{\text {sat }}}{\mathrm{d} T}=3 b\left(\frac{1}{c_{G}^{*}}-\frac{1}{c_{L}^{*}}\right) \frac{P_{c}}{T_{c}} \frac{\mathrm{d} P_{\text {sat }, r}}{\mathrm{~d} T_{r}}
$$

can be rewritten as (remind that $b P_{c}=R T_{c} / 8$ )

$$
\begin{aligned}
\left(V_{G}^{*}-V_{L}^{*}\right) \frac{\mathrm{d} P_{\text {sat }}}{\mathrm{d} T} & =48 b \frac{P_{c}}{T_{c}}\left(1-T_{r}\right)^{1 / 2}+O\left[\left(1-T_{r}\right)^{3 / 2}\right] \\
& =6 R\left(1-T_{r}\right)^{1 / 2}+O\left(1-T_{r}\right)^{3 / 2} .
\end{aligned}
$$

Thus, at near-critical temperature, the enthalpy of vaporization, $L_{v}$, is temperature dependent [see equation (4)],

$$
L_{v}=T\left(V_{G}^{*}-V_{L}^{*}\right) \frac{\mathrm{d} P_{\text {sat }}}{\mathrm{d} T}=6 R T_{C}\left(1-T_{r}\right)^{1 / 2}+O\left[\left(1-T_{r}\right)^{3 / 2}\right] .
$$

Notice that Landau and Lifshiz obtained this same temperature dependence in another way [9]. From equation (6b), for the entropy, we obtain (at $T \approx T_{c}$ )

$$
S_{\mathrm{sat}}\left(T, V_{G}^{*}\right)-S\left(T, V_{L}^{*}\right)=\frac{L_{v}}{T}=6 R\left(1-T_{r}\right)^{1 / 2}+O\left[\left(1-T_{r}\right)^{3 / 2}\right] .
$$

${ }^{1}$ The big $O$ notation or asymptotic notation is a mathematical notation used to describe the asymptotic behavior of functions. Its purpose is to characterize a function's behavior for very large, or very small, inputs in a simple but rigorous way that enables comparison to other functions. An asymptotic expansion is a formal series, which has the property that truncating the series after a finite number of terms provides an approximation to a given function as the argument of the function tends toward a particular, often, finite point. Big $O$ notation (in mathematics) is usually used to characterize the residual term of a truncated infinite series, and especially of an asymptotic series. 
Equation (43) can be obtained from equation (42) noticing that $L_{v}=T\left(S_{2}-S_{1}\right)$ at $T=$ const.

For the free energy we have [see equation (13b)],

$$
\begin{aligned}
F_{\text {sat }}\left(T, V_{G}^{*}\right)-F_{\text {sat }}\left(T, V_{L}^{*}\right) & =-P_{\text {sat }}(T)\left(V_{G}^{*}-V_{L}^{*}\right) \\
& =-12 b P_{c}\left(1-T_{r}\right)^{1 / 2}+O\left\lfloor\left(1-T_{r}\right)^{3 / 2}\right\rfloor \\
& =-\frac{3}{2} R T_{c}\left(1-T_{r}\right)^{1 / 2}+O\left[\left(1-T_{r}\right)^{3 / 2}\right] .
\end{aligned}
$$

For the internal energy [see equation (12b)],

$$
\begin{aligned}
U_{\text {sat }}\left(T, V_{G}^{*}\right)-U_{\text {sat }}\left(T, V_{L}^{*}\right) & =L_{v}-P_{\text {sat }}\left(V_{G}^{*}-V_{L}^{*}\right) \\
& =36 b P_{c}\left(1-T_{r}\right)^{1 / 2}+O\left[\left(1-T_{r}\right)^{3 / 2}\right\rfloor \\
& =\frac{9}{2} R T_{c}\left(1-T_{r}\right)^{1 / 2}+O\left[\left(1-T_{r}\right)^{3 / 2}\right] .
\end{aligned}
$$

Equations (43)-(45) agree with equation $\Delta F=\Delta U-T \Delta S$ obtained from $F=U-T S$ at saturated conditions and $T=$ const.

For the heat capacity at constant volume, we get from equations (15) and (37), and reminding that $T=T_{c} T_{r}$,

$$
\left(\frac{\partial C_{\mathrm{sat}, V}}{\partial V}\right)_{T}=T\left(\frac{\partial^{2} P_{\mathrm{sat}}}{\partial T^{2}}\right)_{V} \approx 9.6 \frac{T}{T_{c}^{2}} P_{c} .
$$

Here, the sign $\approx$ means that number coefficient 9.6 is not exact. After integration of this equation, we obtain

$$
C_{\text {sat }, V}\left(V_{G}^{*}, T\right)-C_{\text {sat }, V}\left(V_{L}^{*}, T\right) \approx 9.6 \frac{T}{T_{c}^{2}} P_{c}\left(V_{G}^{*}-V_{L}^{*}\right), \quad 0.46<T_{r} \leqslant 1 .
$$

This equation can be written, taking into account equations (28), (38), and $b P_{c}=R T_{c} / 8$, as,

$$
C_{\mathrm{sat}, V}\left(V_{G}^{*}, T\right)-C_{\mathrm{sat}, V}\left(V_{L}^{*}, T\right) \approx 9.6 \frac{T}{T_{c}^{2}} 3 b P_{c}\left(1 / c_{G}^{*}-1 / c_{L}^{*}\right) \approx 9.6 \frac{3}{2} R\left(1-T_{r}\right)^{1 / 2} .
$$

Note that $C_{\mathrm{sat}, V}\left(V_{G}^{*}, T\right)$ is equal to the heat capacity of an ideal gas $\left(C_{V}\left(V_{G}^{*}, T\right)=\right.$ $i R / 2$ where $i$ is the number of degrees of freedom of a gas molecule) and $C_{\text {sat }, V}\left(V_{L}^{*}, T\right)$ is the heat capacity of the van der Waals liquid, $C_{V}^{\text {liq }}$. Thus, the expression for $C_{V}^{\text {liq }}$, which does not change at $V_{L}<V_{L}^{*}$, is

$$
C_{V}^{\mathrm{liq}}=\frac{i}{2} R-9.6 \frac{3}{2} R\left(1-T_{r}\right)^{1 / 2} .
$$


Table 1

$C_{\mathrm{P}} / \mathrm{J} \mathrm{K}^{-1} \mathrm{~mol}^{-1}$ values in the solid, liquid, and gas phases [10]. In the third column are the theoretical gas values.

\begin{tabular}{llccl}
\hline Substance & Gas & Gas (theoretical) & Liquid & \multicolumn{1}{c}{ Solid } \\
\hline Aluminum & 21.38 & 20.8 & 24.21 & 24.35 \\
Carbon & 20.838 & 20.8 & & 8.527 (graphite) 6.113 (diamond) \\
Methanol & 43.89 & 33.2 & 81.6 & \\
Ethanol & 65.44 & 33.2 & 111.46 & \\
Benzene & 81.67 & 33.2 & 136.1 & \\
Chlorine & 33.91 & 29.1 & & 36.90 \\
Iodine & 54.44 & 29.1 & & 25.40 \\
Lead & 20.79 & 20.8 & & 27.983 \\
Mercury & 20.786 & 20.8 & & 28.24 \\
Sodium & 20.79 & 20.8 & & 50.50 \\
NaCl & & 29.1 & & 23.6 (monoclin.) 22.64 (rhombic) \\
Sulphur & 23.673 & 20.8 & 75.291 & 37.8 (ice, $0^{\circ} \mathrm{C}$ ) \\
Zinc & 20.79 & 20.8 & & \\
Water & 33.58 & 33.2 & & \\
\hline
\end{tabular}

At the critical temperature, $V_{L}^{*}=V_{G}^{*}, C_{V}\left(V_{G}^{*}, T\right)=C_{V}\left(V_{L}^{*}, T\right)$, and the liquid cannot be distinguished from the gas. At lower temperature, the change of phase from liquid to gas should be considered, and the heat capacity jumps to another value (we are facing a phase transitions of the first order), and we have obtained the value of this jump. Thus, at the phase transition the heat capacity of a liquid is smaller than the heat capacity of a gas. This fact could be explained considering that the translational and rotational motions are relatively frozen in a liquid. In reality, forgetting van der Waals, the heat capacity of a liquid (as well as the heat capacity of a solid) is larger that the heat capacity of a gas (see table 1). The theoretical gas values in table 1 were obtained with equation, $C_{P}=$ $C_{V}+R=R(i+2) / 2$, which is valid for an ideal gas; here $i$ equals the number of degrees of freedom, and only translational and rotational degrees of freedom have been taken into account.

\subsection{Thermodynamic functions at low-temperature limit}

In the other limit, at near zero temperatures, we have from equations (35) and (B4),

$$
P_{\text {sat }}\left(V_{G}^{*}-V_{L}^{*}\right)=3 b P_{c}\left(3-c_{G}^{*}-c_{L}^{*}\right)\left(c_{L}^{*}-c_{G}^{*}\right)=8 b P_{c} \frac{T}{T_{c}}+O\left(T_{r}^{2}\right) .
$$

While from equations (26), (28)-(34) one can obtain

$$
c_{G}^{*}=\frac{81}{8 e T_{r}} \exp \left(-\frac{27}{8 T_{r}}\right) \text {, }
$$




$$
P_{\text {sat }, r}=c_{G}^{*} \frac{3}{2}\left(1+\sqrt{1-\frac{32}{27} T_{r}}\right){ }_{\frac{8}{9}}^{8} T_{r} \cong \frac{8}{3} c_{G}^{*} T_{r}=\frac{27}{e} \exp \left(-\frac{27}{8 T_{r}}\right) .
$$

This result (equation (52)) can also be obtained from the Clausius-Clapeyron equation (4a) if the enthalpy of vaporization, $L_{v}$, is not temperature-dependent and equals $27 R T_{C} / 8$. have

At very low temperatures $\left(T_{r} \rightarrow 0\right)$ and with equations (51) and (52) we

$$
\begin{gathered}
\frac{\mathrm{d} P_{\text {sat }, r}}{\mathrm{~d} T_{r}}=\frac{27 \times 27}{8 e} \frac{1}{T_{r}^{2}} \exp \left(-\frac{27}{8 T_{r}}\right), \\
\frac{\mathrm{d}^{2} P_{\mathrm{sat}, r}}{\mathrm{~d} T_{r}^{2}}=\frac{27 \times 27 \times 27}{64 e} \frac{1}{T_{r}^{4}} \exp \left(-\frac{27}{8 T_{r}}\right) .
\end{gathered}
$$

Notice that, due to the condition $T_{r} \rightarrow 0$, a term has been dropped in equation (54).

$$
\begin{aligned}
\left(V_{G}^{*}-V_{L}^{*}\right) \frac{\mathrm{d} P_{\mathrm{sat}}}{\mathrm{d} T} & =3 b\left(\frac{1}{c_{G}^{*}}-\frac{1}{c_{L}^{*}}\right) \frac{P_{c}}{T_{c}} \frac{\mathrm{d} P_{\mathrm{sat}, r}}{\mathrm{~d} T_{r}} \cong 3 b \frac{P_{c}}{T_{c}} \frac{1}{c_{G}^{*}} \frac{\mathrm{d} P_{\mathrm{sat}, r}}{\mathrm{~d} T_{r}} \\
& =27 b \frac{P_{c}}{T_{c}} \frac{1}{T_{r}}=\frac{27 R}{8 T_{r}} .
\end{aligned}
$$

Thus, at near-zero temperature $\left(\mathrm{T} \rightarrow 0 \mathrm{~K}, b P_{c}=R T_{c} / 8\right)$ we have

$$
L_{v}=T\left(V_{G}^{*}-V_{L}^{*}\right) \frac{\mathrm{d} P_{\text {sat }}}{\mathrm{d} T}=27 b P_{c}=\frac{27}{8} R T_{C},
$$

i.e., the enthalpy of vaporization does not depend on the temperature, and, as it must be, the entropy of a gas is larger than the entropy of a liquid:

$$
S_{\text {sat }}\left(T, V_{G}^{*}\right)-S_{\text {sat }}\left(T, V_{L}^{*}\right)=\frac{L_{v}}{T}=\frac{27 R}{8 T_{r}} .
$$

As WEOS does not take into account quantum effects, equation (57) contradicts the Nernst theorem (third law of thermodynamics): $S(T)$ approaches a finite limit at $T=0$, which does not depend on specifics of processes that brought the system to the $T=0$ state. Notice that the same problem holds for an ideal gas, where $S\left(T, V_{2}\right)-S\left(T, V_{1}\right)=R \ln \left(V_{2} / V_{1}\right)$.

$$
F_{\text {sat }}\left(T, V_{G}^{*}\right)-F_{\text {sat }}\left(T, V_{L}^{*}\right)=-P_{\text {sat }}(T)\left(V_{G}^{*}-V_{L}^{*}\right)=-8 b P_{c} T_{r}=-R T,
$$

i.e., the free energy of a gas is equal to the free energy of a liquid at $T \rightarrow 0$,

$$
U_{\text {sat }}\left(T, V_{G}^{*}\right)-U_{\text {sat }}\left(T, V_{L}^{*}\right)=L_{v}-P_{\text {sat }}\left(V_{G}^{*}-V_{L}^{*}\right)=\frac{27}{8} R T_{C}-R T,
$$


i.e., the internal energy of a gas is larger then the free energy of a liquid. The equations (57)-(59) agree with equation $\Delta F=\Delta U-T \Delta S$ obtained from $F=$ $U-T S$ at saturated conditions and $T=$ const.

The change of the heat capacities at the phase change (liquid $\rightarrow$ gas) is,

$$
C_{\text {sat }, V}\left(V_{G}^{*}, T\right)-C_{\text {sat }, V}\left(V_{L}^{*}, T\right)=\frac{27 \times 27 \times 27}{64 e T_{r}^{2} T} \exp \left(-\frac{27}{8 T_{r}}\right) P_{c}\left(V_{G}^{*}-V_{L}^{*}\right) .
$$

At low temperature, $V_{G}^{*} \gg V_{L}^{*}$, the van der Waals gas behaves as an ideal gas, and the perfect gas law is valid, $P_{\text {sat }} V_{G}^{*}=R T$. Using this circumstances and taking into account equation (52), we obtain

$$
C_{\mathrm{sat}, V}\left(V_{G}^{*}, T\right)-C_{\mathrm{sat}, V}\left(V_{L}^{*}, T\right)=\frac{27 \times 27}{64} \frac{T_{c}^{2}}{T^{2}} R .
$$

That means that the difference of heat capacities of a van der Waals gas and liquid at constant volume and at very low temperatures increases. This result contradicts again the Nernst theorem, according to which, $C_{V}=0$ at $T=0 \mathrm{~K}$. The same contradiction exists for an ideal gas, whose heat capacity does not depend on the temperature and it equals $i R / 2$. If we were to use for the heat capacity of a van der Waals gas the classical value $C_{G}^{\text {sat }}\left(V_{G}^{*}, T\right)=i R / 2$ (at low temperature the van der Waals gas behaves as an ideal gas), we would obtain, using equation (61) (for $V<V_{L}^{*}$ ), that the heat capacity of a van der Waals liquid $C_{V}^{\text {liq }}$ can be negative. That means that the van der Waals equation (as any classical theory that does not take into account quantum effects. Some of these effects have recently been treated in [11]) gives no correct (even qualitative) results for the heat capacities at very low temperatures.

Note that for real substances at $T \rightarrow 0$, there is no liquid-vapor transition, there is only solid-vapor transition (see figure 4); for water, solid-liquid transition exist also at $T<T_{c}$. Thus, equations obtained at the near-zero temperature could only be applied in the temperature interval $T_{\text {triple point }}<T \ll T_{c}$ (WEOS describes liquid-vapor phase transition only). That means that these equations can be used for substances with $T_{\text {triple point }} \ll T_{c}$. For a list of critical temperatures and triple point temperatures see table 2 . It can be seen that for real substances, the inequality $T_{\text {triplepoint }} \ll T_{c}$ is never fulfilled.

\section{Conclusions}

The equations for reduced liquid and gas concentrations at saturated conditions, for the changes of enthalpy of vaporization, entropy, Helmholtz function, Gibbs function, and the heat capacity at constant volume of a van der Waals gas (liquid) during the phase transition were obtained in two different limit approximations. The first limit approximation is at the near-critical temperatures, i.e., for $T \rightarrow T_{c}$, the other limit approximation is at the near-zero temperatures, $T \rightarrow 0$. 


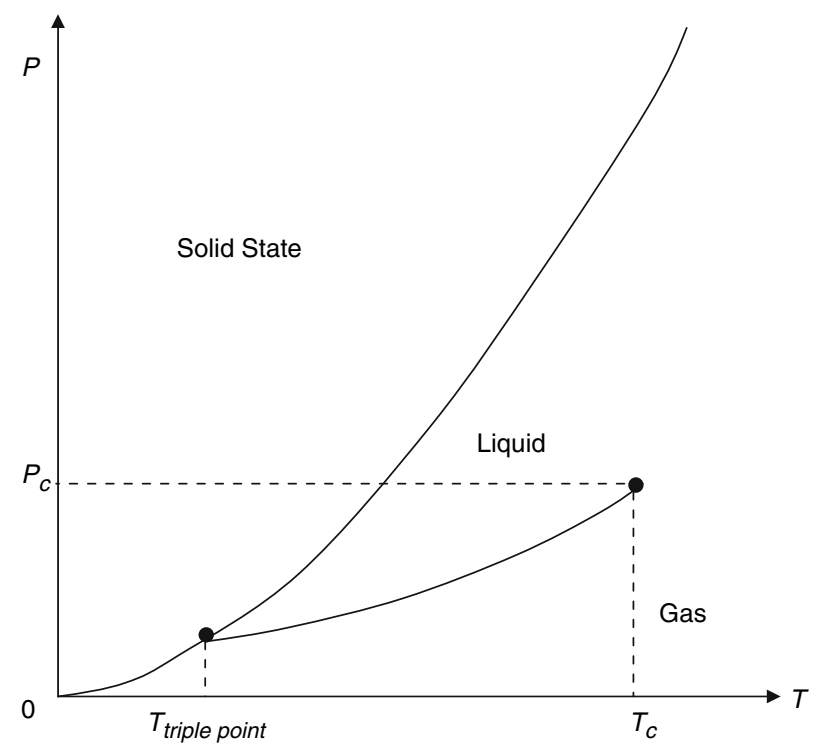

Figure 4. A nonspecific phase diagram, which resembles more to a phase diagram for carbon dioxide than to a phase diagram for water, where the solid-liquid curve has a negative slope. For sheer curiosity $\mathrm{CO}_{2}$ has its triple point at $\mathrm{T}=216.8 \mathrm{~K}$ and $\mathrm{P}=5.11$ bar.

Table 2

Critical temperatures, $T_{c}$, and triple point temperatures, $T_{t}[10,12-14]^{*}$.

\begin{tabular}{|c|c|c|}
\hline Substance & $T_{c} / \mathrm{K}$ & $T_{t} / \mathrm{K}$ \\
\hline Hydrogen, $\mathrm{H}_{2}$ & 32.98 & 13.81 \\
\hline Helium, ${ }^{3} \mathrm{He}$ & 3.35 & - \\
\hline Helium, ${ }^{4} \mathrm{He}$ & 5.1953 & $2.18(\lambda$ point \\
\hline Nitrogen, $\mathrm{N}_{2}$ & 126.19 & 63.2 \\
\hline Oxygen, $\mathrm{O}_{2}$ & 154.58 & 54.4 \\
\hline $\mathrm{Neon}, \mathrm{Ne}$ & 44.49 & 24.56 \\
\hline Argon, Ar & 150.7 & 83.8 \\
\hline Methane, $\mathrm{CH}_{4}$ & 190.56 & 88.7 \\
\hline Ammonia, $\mathrm{NH}_{3}$ & 405.40 & 195.49 \\
\hline Water, $\mathrm{H}_{2} \mathrm{O}$ & 647.4 & 273.16 \\
\hline Carbon dioxide, $\mathrm{CO}_{2}$ & 304.21 & 216.6 \\
\hline $\mathrm{Hg}$ & $>1800$ & 234.3156 \\
\hline
\end{tabular}

* The most recent data have been chosen.

Although the heat capacities at constant volume of a van der Waals gas and liquid do not depend on volume, these heat capacities have different values and their change during the phase transition was calculated. It was obtained that at near-critical temperatures, the heat capacity of a gas is larger than the heat capacity of a liquid, and the difference between these heat capacities decreases to zero 
as $\left(1-T / T_{c}\right)^{1 / 2}$ when the temperature increases and approaches to critical temperature.

Results obtained at near-zero temperatures are interesting only from a theoretical point of view because for real substances, the obtained equations are only valid in the temperature interval $T_{\text {triple point }}<T \ll T_{c}$, i.e., for substances with $T_{\text {triple point }} \ll T_{c}$. Such substances are, actually, missing.

\section{Appendix A: solution at near-critical temperatures $\left(T_{r} \rightarrow 1, c_{L}^{*} \rightarrow 1, c_{G}^{*} \rightarrow 1\right)$}

To solve equation (25) parametrically let us introduce the new variables, $x_{+}$ and $x_{-}$[5], which allow rewriting equation (25) as equation (A3)

$$
\begin{gathered}
x_{+}=\frac{1}{3 / c_{L}^{*}-1}, \\
x_{-}=\frac{1}{3 / c_{G}^{*}-1} . \\
\ln \frac{x_{+}}{x_{-}}=\frac{\left(x_{+}-x_{-}\right)\left(x_{+}+x_{-}+2\right)}{2 x_{+} x_{-}+x_{+}+x_{-}} .
\end{gathered}
$$

Setting both sides of this equation equal to $2 y$ the solution (A5) is obtained

$$
\begin{gathered}
\ln \frac{x_{+}}{x_{-}}=2 y, \\
x_{+}=\mathrm{e}^{2 y} x_{-}, \text {and } x_{-}=\mathrm{e}^{-2 y} x_{+} .
\end{gathered}
$$

The final result could be written as: $x_{ \pm}=\mathrm{e}^{ \pm y} f(y)$, with $f(y)$ given by,

$$
f(y)=\frac{y \cosh y-\sinh y}{\sinh y \cosh y-y} .
$$

Or

$$
x_{+}=\frac{2 \mathrm{e}^{y} y\left(\mathrm{e}^{-y}+\mathrm{e}^{-y}\right)-\mathrm{e}^{y}+a^{-y}}{\mathrm{e}^{2 y}-\mathrm{e}^{-2 y}-4 y} .
$$

The equation for $x_{-}$is obtained from the inspection of equations (A5) and (A7). Taking into account equations (A1) and (A2) we have

$$
c_{L}^{*}=\frac{3}{1+1 / x_{+}}=\frac{3}{1+\frac{1}{2} \mathrm{e}^{-y} \frac{\mathrm{e}^{2 y}-\mathrm{e}^{-2 y}-4 y}{y\left(\mathrm{e}^{y}+\mathrm{e}^{-y}\right)-\mathrm{e}^{y}+\mathrm{e}^{-y}} .}
$$


At the near critical temperature, $y \ll 1$, and with the help of Mathematica [15] the following series can be written,

$$
c_{L}^{*}=1+\frac{2}{3} y+\frac{2}{45} y^{2}-\frac{8}{135} y^{3}+\frac{2}{576} y^{4}+\frac{272}{42525} y^{5}+\frac{4}{18225} y^{6}+O\left(y^{7}\right) .
$$

By the same way, one can obtain the following two equations for $c_{G}^{*}$,

$$
\begin{gathered}
c_{G}^{*}=\frac{3}{1+1 / x_{-}}=\frac{3}{1+\frac{1}{2} \mathrm{e}^{y} \frac{\mathrm{e}^{2 y}-\mathrm{e}^{-2 y}-4 y}{y\left(\mathrm{e}^{y}+\mathrm{e}^{-y}\right)-\mathrm{e}^{y}+\mathrm{e}^{-y}}}, \\
c_{G}^{*}=1-\frac{2}{3} y+\frac{2}{45} y^{2}+\frac{8}{135} y^{3}-\frac{2}{576} y^{4}-\frac{272}{42525} y^{5}+\frac{4}{18225} y^{6}+O\left(y^{7}\right) .
\end{gathered}
$$

Let us now take equation (27)

$$
\frac{1}{8}\left(c_{G}^{*}+c_{L}^{*}\right)\left(3-c_{G}^{*}\right)\left(3-c_{L}^{*}\right)=T_{r} .
$$

Inserting the series (A9) and (A11) into equation (A12), we obtain an equation with precision till $O\left(y^{7}\right)$

$$
1-\frac{1}{9} y^{2}+\frac{1}{75} y^{4}-\frac{946}{63785} y^{6}=1-\left(1-T_{r}\right)
$$

Introducing the new variable,

$$
x^{2}=1-T_{r}
$$

we obtain from equation (A13), with precision till $y^{4}$ (inclusive),

$$
1-\frac{1}{9} y^{2}+\frac{1}{75} y^{4}=1-x^{2}
$$

The solution of this equation is

$$
y^{2}=\frac{25-\sqrt{625-2700 x^{2}}}{6} .
$$

With precision till $x^{5}$, we obtain

$$
y^{2}=9 x^{2}+\frac{243}{25} x^{4}+O\left(x^{5}\right)
$$

or

$$
y=3 x\left(1+\frac{27}{50} x^{2}\right)+O\left(x^{4}\right)
$$


Let us introduce this equation into formula (A9). With precision till $y^{4}$ (or $x^{4}$ ) we obtain

$$
\begin{aligned}
c_{L}^{*} & =1+\frac{2}{3} y+\frac{2}{45} y^{2}-\frac{8}{135} y^{3}+O\left(y^{4}\right) \\
& =1+\frac{2}{3}\left[3 x\left(1+\frac{27}{50} x^{2}\right)\right]+\frac{2}{45}(3 x)^{2}-\frac{8}{135}(3 x)^{3}+O\left(x^{4}\right) \\
& =1+2 x+\frac{2}{5} x^{2}-\frac{13}{25} x^{3}+O\left(x^{4}\right) .
\end{aligned}
$$

Comparing equations (A9) and (A8) for $c_{L}^{*}$ and $c_{G}^{*}$, we immediately have,

$$
c_{G}^{*}=1-2 x+\frac{2}{5} x^{2}+\frac{13}{25} x^{3}+O\left(x^{4}\right) .
$$

From the fitting procedure, we got that with precision better than $1 \%$,

$$
\begin{gathered}
c_{L}^{*}=1+2 x+\frac{2}{5} x^{2}-\frac{13}{25} x^{3}+0.115 x^{4}, \\
c_{G}^{*}=1-2 x+\frac{2}{5} x^{2}+\frac{13}{25} x^{3}+0.207 x^{4}, \\
\frac{1}{2}\left(c_{L}^{*}+c_{G}^{*}\right)=1+\frac{2}{5} x^{2}+0.161 x^{4} .
\end{gathered}
$$

Equation (A21) is valid for $0 \leqslant T_{r} \leqslant 1$ (always! see figure 1), equation (A22) is valid for $0.46<T_{r} \leqslant 1$ (see figure 1), and equation (A23) is valid for $0.25<T_{r} \leqslant 1$ (see figure 2). These validity limits, as well as the coefficients 0.115 and 0.207 in equations (A21) and (A22), were obtained by fitting the numerical calculations and equations (A21) and (A22).

\section{Appendix B: solution at near-zero temperatures $\left(T_{r} \rightarrow 0, c_{L}^{*} \rightarrow 3, c_{G}^{*} \rightarrow 0\right)$}

(i) To obtain an equation for $c_{L}^{*}$ in this limit, let us use equation (25) from [7] [here equation (27)],

$$
\frac{1}{8}\left(c_{G}^{*}+c_{L}^{*}\right)\left(3-c_{G}^{*}\right)\left(3-c_{L}^{*}\right)=T_{r} .
$$

If $T_{r} \rightarrow 0, c_{G}^{*} \rightarrow 0$ and equation (1) takes the form

$$
\frac{3}{8} c_{L}^{*}\left(3-c_{L}^{*}\right)=T_{r}
$$


The solution of this equation is

$$
c_{L}^{*}=\frac{3}{2}\left(1+\sqrt{1-\frac{32}{27} T_{r}}\right) .
$$

Comparison with the numerical calculations follows that equation (B3) is valid with precision better $1 \%$ at $0 \leqslant T_{r}<0.7$ (see figure 1, dashed line). Expressing equation (B3) as a second order series in $T_{r}$, we notice that the following equation is valid with a precision better than $1 \%$ throughout the interval $0 \leqslant$ $T_{r}<0.46$

$$
c_{L}^{*}=3-\frac{8}{9} T_{r}-0.3 T_{r}^{2} .
$$

Coefficient 0.3 was obtained by fitting equation (B4) with numerical calculations.

(ii) To obtain an equation for $c_{G}^{*}$ in the limit $T_{r} \rightarrow 0$, we shall use equation (23) of [7] [here equation (25)]

$$
\ln \frac{3 / c_{G}^{*}-1}{3 / c_{L}^{*}-1}=\frac{c_{L}^{*}-c_{G}^{*}}{c_{L}^{*}+c_{G}^{*}}\left(\frac{3}{3-c_{L}^{*}}+\frac{3}{3-c_{G}^{*}}\right) .
$$

The numerical calculations allows to obtain that $c_{G}^{*}<2 \times 10^{-3}$ and $c_{L}^{*}>2.6$ if $T_{r}<0.34$. At these temperatures, equation (B5) can be rewritten as

$$
\ln \frac{3 / c_{G}^{*}-1}{3 / c_{L}^{*}-1}=\left(\frac{3}{3-c_{L}^{*}}+1\right) \text {. }
$$

Or,

$$
\frac{3}{c_{G}^{*}}=1+\left(\frac{3}{c_{L}^{*}}-1\right) \exp \left(\frac{3}{3-c_{L}^{*}}+1\right) .
$$

Equation (B7) can be obtained thanks to the following series of rearrangements, $\frac{3 / c_{G}^{*}-1}{3 / c_{L}^{*}-1}=\exp \left(\frac{3}{3-c_{L}^{*}}+1\right) \rightarrow \frac{3}{c_{G}^{*}}-1=\left(\frac{3}{c_{L}^{*}}-1\right) \exp \left(\frac{3}{3-c_{L}^{*}}+1\right)$. Due to the exponential term $\left(c_{L}^{*} \approx 3\right)$, the unity in right-hand side of equation (B7) is small in comparison with the second right-hand term. Now, as, $\frac{3}{c_{G}^{*}}=$ $\left(\frac{3}{c_{L}^{*}}-1\right) \exp \left(\frac{3}{3-c_{L}^{*}}+1\right)$, equation (B8) is retrieved,

$$
c_{G}^{*}=\frac{3 c_{L}^{*}}{e\left(3-c_{L}^{*}\right)} \exp \left(-\frac{1}{1-c_{L}^{*} / 3}\right) .
$$

Introducing the equation (B3) into equation (B8), we obtain the dependence of $c_{G}^{*}$ on $T_{r}$ in the low temperature limit. Comparison with the numerical calculations shows that equation (B8) together with equation (B3) is valid with an error smaller than $1 \%$ at $0 \leqslant T_{r}<0.33$ (see figure 2 , dashed line). 
(iii) As the concentration of gas is very small at the low temperature limit, then, $\frac{1}{2}\left(c_{L}^{*}+c_{G}^{*}\right) \approx \frac{1}{2} c_{L}^{*}$. Thus, from equation (B4), we get,

$$
\frac{1}{2}\left(c_{L}^{*}+c_{G}^{*}\right)=\frac{3}{2}-\frac{4}{9} T_{r}-0.15 T_{r}^{2} .
$$

Comparison with the numerical calculations shows that this equation is valid with an error smaller than $1 \%$ at $0 \leqslant T_{r}<0.64$ (see figure 3 , dashed line).

\section{References}

[1] J. Wisniak and M. Golden, J. Chem. Educ. 75 (1998) 200.

[2] J.G. Eberhart, J. Chem. Educ. 66 (1989) 906.

[3] L. Pogliani, J. Chem. Educ. 83 (2006) 155.

[4] S.I. Sandler, Chemical and Engineering Thermodynamics, 3rd ed. (Wiley, New York, 1999).

[5] J. Wisniak, Chem. Educ. 5 (2000) 149.

[6] J. Lekner, Am. J. Phys. 50 (1982) 161.

[7] M.N. Berberan-Santos, E.N. Bodunov and L. Pogliani, Am. J. Phys. 70 (2002) 438.

[8] C.F. Bohren and B.A. Albreht, Atmospheric thermodynamic (Oxford University Press, New York, 1998).

[9] E.M. Lifshiz and L.D. Landau, Statistical Physics, 3rd ed., Vol. 5, Part 1, Eq. (152.15) (Butterworth-Heinemann, London 1984).

[10] P.W. Atkins, Physical Chemistry, 5th ed. (Oxford, Oxford, 1994) p. 1061.

[11] M.N. Berberan-Santos, E.N. Bodunov and L. Pogliani, J. Math. Chem. 37 (2005) 101.

[12] M.W. Zemansky, R.H. Dittman, Heat and Thermodynamics (McGraw-Hill, New York, 1981) p. 31.

[13] International temperature scale of 1990, http: //en.wikipedia.org/wiki//International_Tempera ture_Scale_of_1990 (accessed October 2006).

[14] Teragon's summary of cryogenic properties (updated 02/2005), http://www.trgn. com/database/cryogen.html (accessed October 2006).

[15] Mathematica 5.0, Wolfram Resarch, http://www.wolfram.com. 